\title{
CONDITIONS UNDER WHICH DISKS ARE P-LIFTABLE
}

\author{
BY
}

\section{EDYTHE P. WOODRUFF(1)}

ABSTRACT. A generalization of the concept of lifting of an n-cell is studied. In the usual upper semicontinuous decomposition terminology, let $S$ be a space, $S / G$ be the decomposition space, and the projection mapping be $P: S \rightarrow$ $S / G$. A set $X^{\prime} \subset S$ is said to be a $P$-lift of a set $X \subset S / G$ if $X^{\prime}$ is homeomorphic to $X$ and $P\left(X^{\prime}\right)$ is $X$.

Examples ate given in which the union of two $P$-liftable sets does not $P$-lift. We prove that if compact 2-manifolds $A$ and $B$ each $P$-lift, their union is a disk in $E^{3} / G$, their intersection misses the singular points of the projection mapping, and the intersection of the singular points with the union of $A$ and $B$ is a 0 dimensional set, then the union of $A$ and $B$ does $P$-lift.

Even if a disk $D$ does not $P$-lift, it is proven that for $\epsilon>0$ there is a $P$-liftable disk $\epsilon$-homeomorphic to $D$, provided that the given decomposition of $E^{3}$ is either definable by 3 -cells, or the set of nondegenerate elements is countable and $E^{3 / G}$ is homeomorphic to $E^{3}$. With further restrictions on the decomposition, this $P$-liftable disk can be chosen in such a manner that it agrees with $D$ on the singular points of $D$.

1. Introduction. A generalization of the concept of lifting of an n-cell is studied. In the usual upper semicontinuous decomposition terminology, let $S$ be a space, $S / G$ be the decomposition space, and the projection mapping be $P: S$ $\rightarrow S / G$. In the standard definition (McAuley [9]) a lift of an $n$-cell $a \subset S / G$ is an $n$-cell $\beta \subset S$ such that $\beta$ maps homeomorphically onto $a$ under the projection mapping $P$. The generalization is defined as follows: A set $X^{\prime} \subset S$ is said to be a $P$-lift of a set $X \subset S / G$ if $X^{\prime}$ is homeomorphic to $X$ and $P\left(X^{\prime}\right)=X$. Notice that a point in $X$ may be the image of a nondegenerate set in $X^{\prime}$.

In $\S 2$ we investigate whether $X \cup Y$ has a $P$-lift when $X$ and $Y$ each $P$-lift. Examples are given in which $X \cup Y$ does not $P$-lift. Additional hypotheses can

Presented to the Society, September 3, 1971 under the title Disks in $E^{3 / G}$; received by the editors September 23, 1971 and, in revi sed form, October 5, 1972

AMS (MOS) subject classifications (1970). Primary 54B15, 54B99, 54C10, 57A10, 57A40; Secondary 57A60, 57A30.

Key words and phrases. Decomposition space, topology of $E^{3}$, lift of a space, $P$. lift, monotone decomposition, cellular decomposition, decomposition definable by 3 -cells, tame, neighborhoods of submanifolds.

(1) This paper represents a portion of the author's Ph.D. dissertation written under the direction of Louis F. McAuley and presented to the faculty of the State University of New York at Binghamton. 
be imposed to assure that $X \cup Y$ will $P$-lift. One such theorem states: Suppose that $A$ and $B$ are compact 2-manifolds that each $P$-lift; $A \cup B$ is a disk $D C$ $E^{3} / G ; A \cap B \cap P(H)=\varnothing$; and $(A \cup B) \cap P(H)$ is 0-dimensional. Then $A \cup B$ $P$-lifts.

It is easy to construct a decomposition $G$ of $E^{3}$ such that there is a disk $D$ $C E^{3} / G$ that is not the image under projection of any disk in $E^{3}$. This implies that $D$ does not $P$-lift. Consider, for example, the set $R$ obtained by rotating the following around the $z$-axis: $\mathrm{Cl}\{(x, z) \mid z=\sin 1 / x, 0<x \leq 1\}$. This set $R$ is not a disk because it is not locally connected in the plane on the segment $s=\{(0, z) \mid$ $-1 \leq z \leq 1\}$. If we use the decomposition of $E^{3}$ in which $s$ is the only nondegenerate element, then the image of the set $R$ is a disk $D$, but $R$ does not contain any disk that projects onto $D$. This condition is not true for all disks homeomorphically close to $D$. There are disks that agree with $R$ except very close to $s$ and do project onto disks. In $\$ 3$ hypotheses are studied that assure that even when a disk does not $P$-lift, homeomorphically close to it there is a disk that does $P$-lift. It is noted that there exists an example of a 0 -dimensional, pointlike decomposition of $E^{3}$ such that for a particular disk $D \subset E^{3 / G}$ and $\epsilon>0$, there is no $P$-liftable disk $D_{\epsilon}$ that is $\epsilon$-homeomorphic to $D$.

In $\$ 4$ we show that with certain hypotheses on the decomposition $G$ of $E^{3}$, arbitrarily close to a given disk $D$ it is not only possible to find a $P$-liftable disk $D_{\epsilon}$, but the disk $D_{\epsilon}$ can be made to agree with $D$ on the singular points of the projection $P$.

Notations. Throughout the paper we use $\mathrm{Cl}$ to denote set closure and Int to denote set interior. The distance between points $p$ and $q$ is denoted by $d(p, q)$. Each decomposition $G$ will be assumed to be upper semicontinuous. The symbol $H$ denotes the set of nondegenerate elements of $G$, and $H^{*}$ is the union of the elements of $H$. We use $P$ to denote the natural projection mapping of $E^{3}$ onto $E^{3} / G$.

2. Conditions under which the union of two sets $P$-lifts. The following two examples show that it is not necessarily true that if two sets each $P$-lift, then their union will $P$-lift.

Example 1. Let $\left\{S_{i}\right\}$ be the sequence of nested topological 2-spheres:

$$
\begin{aligned}
S_{i}= & \{(x, y, z)|| x|\leq 1 / 2+1 / i,| y|\leq 1 / 2+1 / i,| z \mid=1 / i\} \\
& \cup\{(x, y, z)|| x|\leq 1 / 2+1 / i,| y|=1 / 2+1 / i,| z \mid \leq 1 / i\} \\
& \cup\{(x, y, z)|| x|=1 / 2+1 / i,| y|\leq 1 / 2+1 / i,| z \mid \leq 1 / i\} .
\end{aligned}
$$

From the top face (parallel to the $x y$-plane) of each $S_{i}$ remove the interior of a disk, $D_{i}$, with diameter $1 / 10$. If $i$ is odd, suppose $D_{i}$ is in the left third of the top face of $S_{i}$, and if $i$ is even, suppose $D_{i}$ is in the right third. Let 
$g_{i}=S_{i}-$ Int $D_{i}$. Let $g_{0}$ be the limiting disk $\{(x, y, z)|| x|\leq 1 / 2| y \mid, \leq 1 / 2, z=0\}$. Let the nondegenerate elements of our decomposition $G$ be $H=\left\{g_{0}, g_{1}, g_{2}, \ldots\right\}$. Now, in $E^{3}$ there is a copy $C$ of the set $\{(u, v) \mid v=\sin 1 / u, 0<u \leq 1\} \times[0,1]$ such that $C \cap H^{*}=\varnothing$ and the limiting set of $C$ is in $g_{0}$. (One can think of $C$ as threading through the holes in the $g_{i}{ }^{\prime}$ s.) Since $C$ is homeomorphic to a disk minus a boundary point and $P$ is a homeomorphism on $C$, the set $P(C) \cup P\left(g_{0}\right)$ is a disk in $E^{3} / G$. For our counterexample, we use $A=P(C)$ and $B=P\left(g_{0}\right)$. Then $A \cup B$ does not $P$-lift, though each of $A$ and $B$ does.

Example 2. Let the set $A^{\prime}$ be the union of the following four squares:

$\{(x, y, z) \mid 0 \leq x \leq 1,0 \leq y \leq 1, z=0\}$,

$\{(x, y, z) \mid 0 \leq x \leq 1,-1 \leq y \leq 0, z=1\}$,

$\{(x, y, z) \mid x=0,0 \leq y \leq 1,0 \leq z \leq 1\}$, and

$\{(x, y, z) \mid 0 \leq x \leq 1, y=0,0 \leq z \leq 1\}$.

Let the set $B^{\prime}$ be the union of the following four squares:

$\{(x, y, z) \mid-1 \leq x \leq 0,0 \leq y \leq 1, z=1\}$,

$\{(x, y, z) \mid-1 \leq x \leq 0,-1 \leq y \leq 0, z=0\}$,

$\{(x, y, z) \mid x=0,-1 \leq y \leq 0,0 \leq z \leq 1\}$, and

$\{(x, y, z) \mid-1 \leq x \leq 0, y=0,0 \leq z \leq 1\}$.

Let the only nondegenerate element of the decomposition be the segment $g=$ $\{(x, y, z) \mid x=0, y=0,0 \leq z \leq 1\}$. Each of $A^{\prime}, P\left(A^{\prime}\right), B^{\prime}, P\left(B^{\prime}\right)$, and $P\left(A^{\prime} \cup B^{\prime}\right)$ is a topological disk, but $A^{\prime} \cup B^{\prime}$ is not because it contains the four squares that have the segment $g$ as a common side and are mutually disjoint off $g$. The disk $P\left(A^{\prime} \cup B^{\prime}\right)$ has no $P$-lift.

We will now prove two theorems concerning when a disk that is the union of two $P$-liftable sets has a $P$-lift.

Theorem 1. Suppose that $A$ and $B$ are compact 2-manifolds, $A \cup B$ is a disk in $E^{3} / G$, and $A \cap B \cap P(H)=\varnothing$. Suppose that $A$ and $B$ bave particular $P$-lifts $A^{\prime}$ and $B^{\prime}$, respectively, such that $P \mid A^{\prime}$ and $P \mid B^{\prime}$ are monotone. Then $A \cup B$ P.lifts.

We will first prove a lemma.

Lemma 1.1. Assume the bypotheses of Theorem 1 are true. Let $G_{A^{\prime}}$ denote $\left\{g \cap A^{\prime} \mid g \in G\right\}$ and $H_{A^{\prime}}$, be the set of nondegenerate elements in $G_{A^{\prime}}$. Then no element of $H_{A^{\prime}}$ separates $A^{\prime}$.

Proof. Suppose there is a $g \in H_{A^{\prime}}$ such that $g$ separates $A^{\prime}$. Let $A_{1}^{\prime} \cup$ $A_{2}^{\prime}=A^{\prime}-g$, where $A_{1}^{\prime}$ and $A_{2}^{\prime}$ are separated sets. The sets $A_{1}^{\prime}$ and $A_{2}^{\prime}$ are closed in $A^{\prime}-g$ by the definition of separated. Let $A_{1}=P\left(A_{1}^{\prime}\right)$ and $A_{2}=$ $P\left(A_{2}^{\prime}\right)$. Then $A_{1}$ and $A_{2}$ are closed in $A-P(g)$, since the natural map is closed. Hence, if $A_{1} \cup A_{2}$ is connected, then there must exist a point $p \in A_{1} \cap A_{2}$. 
This implies that $P^{-1}(p) \cap A_{1}^{\prime}$ and $P^{-1}(p) \cap A_{2}^{\prime}$ are each nonempty, and, hence $P^{-1}(p)$ is an element of $H_{A}$, that is not connected. This contradicts the hypothesis. Hence, it must be that $A_{1}$ and $A_{2}$ are separated sets such that $A_{1} \cup A_{2}=$ $A-P(g)$. Now, since $A \cup B$ is a disk, $P(g)$ must have a neighborhood $N$ in $A$ $\cup B$ homeomorphic to the plane or to the closed upper half plane. The set $N$ $P(g)=N \cap\left(A_{1} \cup A_{2} \cup B\right)$ is connected. Since $N$ can have an arbitrarily small diameter, $P(g)$ must be a limit point of $B$. The fact that $B$ is closed implies that $P(g) \in B$. Hence, $P(g) \in A \cap B$. Now, this implies that $P(g) \cap A \cap B$ is nonempty, which contradicts a hypothesis and proves the lemma.

Proof of Theorem 1. The definition of $P$-lift gives us the following diagram:

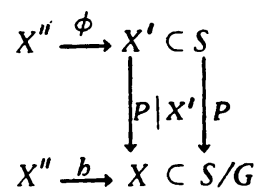

where $X^{\prime \prime}$ is a copy of $X$ and $\phi$ and $b$ are homeomorphisms. Because we are given that $A^{\prime}$ and $B^{\prime}$ are $P$-lifts, the following diagrams with the appropriate definitions do exist:

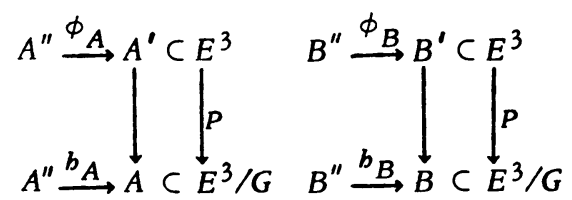

Let us define the set $(A \cup B)^{\prime}$ to be $A^{\prime} \cup B^{\prime}$ for the particular $A^{\prime}$ and $B^{\prime}$ given in the hypothesis. Then $P$ takes $(A \cup B)^{\prime}$ onto $A \cup B$. To know that $(A \cup B)^{\prime}$ is the $P$-lift of $A \cup B$, we must prove that $A \cup B$ and $(A \cup B)^{\prime}$ are homeomorphic. This will be done by defining a set $(A \cup B)^{n}$ that is homeomorphic to $(A \cup B)^{\prime}$ and then considering a decomposition of $(A \cup B)^{\prime \prime}$.

We first wish to consider the set that maps onto $A \cap B$ in the second and third diagrams. Since $A$ and $B$ are compact, the hypothesis $A \cap B \cap P(H)=\varnothing$ implies that $P^{-1}$ is a homeomorphism on $A \cap B$. Hence, $A \cap B$ lifts by McAuley's definition of lift. Since no other points in $E^{3}$ map onto $A \cap B$ by $P$, this lift is the only possible preimage of $A \cap B$. Therefore, it must be the $P$-lift of this set in each of the given diagrams. Therefore, the subset $A \cap B$ of $A$ gives us the following restricted diagram, in which $(A \cap B)_{A}^{\prime}$ and $(A \cap B)_{A}^{\prime \prime}$ are the appropriate subsets from the diagram for $A$.

$$
\begin{aligned}
& A^{\prime \prime} \supset(A \cap B)_{A}^{\prime \prime} \stackrel{\phi_{A} \mid(A \cap B)_{A}^{\prime \prime}}{\longrightarrow}(A \cap B)_{A}^{\prime} \subset A^{\prime} \subset E^{3}
\end{aligned}
$$

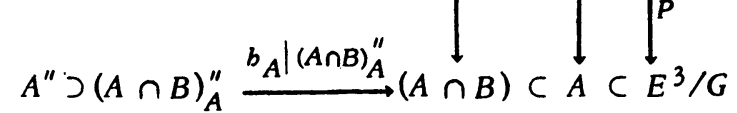


There is a similar diagram for $A \cap B$ considered as a subset of $B$. By the above argument, we know that the subsets $(A \cap B)_{A}^{\prime}$ of $A^{\prime}$ and $(A \cap B)_{B}^{\prime}$ of $B^{\prime}$ are identical.

Define $(A \cup B)^{\prime \prime}$ to be the adjunction space $A^{\prime \prime} \cup B^{\prime \prime}$ for the map $f=\phi_{B}^{-1} \phi_{A}$. Since $f$ is a homeomorphism, the definition of adjunction space implies that $(A \cup B)^{\prime \prime}$ is homeomorphic to $(A \cup B)^{\prime}$. Notice that in $(A \cup B)^{\prime \prime}$ the subsets $A^{\prime \prime}$, $B^{\prime \prime}$, and $(A \cap B)^{\prime \prime}$ are homeomorphic to $A, B$, and $A \cap B$, respectively, but at this point in our proof we do not know that there exist three corresponding homeomorphisms that agree on $(A \cap B)^{\prime \prime}$.

- Because $A$ is contained in a disk and $A^{\prime \prime}$ is homeomorphic to $A$, there is an imbedding $i: A^{\prime \prime} \rightarrow E^{2}$. We will now show that for $g \in H_{A}$, the set $i \phi_{A}^{-1}(g)$ does not separate $E^{2}$. Lemma 1.1 implies that $i \phi_{A}^{-1}(g)$ does not separate $i \phi_{A}^{-1}\left(A^{\prime}\right)$. Hence, if $i \phi_{A}^{-1}(g)$ does separate $E^{2}$, then $i \phi_{A}^{-1}(g)$ must be a component of $i\left(\mathrm{Bd} A^{\prime \prime}\right)$. This implies that $g$ is a component of $\mathrm{Bd} A^{\prime}$. Note that there are the same number of components in $\mathrm{Bd} A^{\prime}$ and $\mathrm{Bd} A$. Now, if one component of $\mathrm{Bd} A^{\prime}$ goes to the point $P(g)$, there must be a set $S$ in Int $A^{\prime}$ that goes onto a component of $(\mathrm{Bd} A)-P(g)$. Now the montone hypothes is can be used to show that $S$ is connected and does not separate Int $A^{\prime}$. Since $S \subset$ Int $A^{\prime}$, the set $S$ must be simply connected. Again using the monotone hypothesis, it can be shown that $S$ cannot map onto a simple closed curve or a simple closed curve minus the point $P(g)$. But this contradicts the assumption that $P(S)$ is a component ( $\mathrm{Bd} A)-$ $P(g)$. Hence, $i \phi_{A}^{-1}(g)$ does not separate $i \phi_{A}^{-1}\left(A^{\prime}\right)$. Also, note that $i \phi_{A}^{-1}(g)$ is connected, because each element of $H_{A^{\prime}}$ is connected.

We now define a decomposition $G_{a}$ of $E^{2}$ such that the elements are points of $E^{2}-i\left(A^{\prime \prime}\right)$ and the sets $i \phi^{-1}(g)$ for each $g \in H_{A^{\prime}}$. This decomposition $G_{a}$ of $E^{2}$ satisfies the hypotheses of the following theorem of Moore [11]:

Theorem. If $G$ is an upper semicontinuous decomposition of the Euclidean plane $E^{2}$ into compact continua such that no element of $G$ separates. $E^{2}$, then the decomposition space associated with $G$ is homeomorphic to $E^{2}$.

This theorem implies that $E^{2} / G_{a}$ is homeomorphic to $E^{2}$.

The following recent result of Siebenmann [13] is stated here in the 2-dimensional case without boundary:

Theorem. Suppose that $P: X \rightarrow X / G$ where $X$ and $X / G$ are 2-manifolds without boundary and $P$ is a $C E$ map. Then $G$ is shrinkable (McAuley).

Recall that McAuley's definition [8] of shrinking requires that, given $\epsilon>0$, there exists a homeomorphism $f_{\epsilon}$ that (1) shrinks each nondegenerate element to a diameter less than $\epsilon$, (2) does not move nondegenerate elements far with respect to a given open covering, and (3) agrees with a given homeomorphism $b$ off a 
given open set containing the nondegenerate elements. In Siebenmann's theorem a $C E$ map is a cell-like map, which means that it is a map $f: X \rightarrow Y$ such that for each point $y \in Y, f^{-1}(y)$ is a compactum that can be imbedded in some Euclidean space as a cellular set. The projection of $E^{2}$ onto $E^{2} / G_{a}$ satisfies this. Since the decomposition $G_{a}$ of $E^{2}$ now satisfies Siebenmann's theorem, it is shrinkable.

The procedure used in defining the decomposition $G_{a}$ from $A, A^{\prime}$, and $A^{\prime \prime}$ has an analogue for $B, B^{\prime}$, and $B^{\prime \prime}$. Hence, the analogous decomposition $G_{b}$ of $E^{2}$ is shrinkable.

The adjunction space $(A \cup B)^{\prime \prime}$ has a decomposition induced by that of $(A \cup B)^{\prime}$. Let $G_{(A \cup B)^{\prime \prime}}$ denote this decomposition of $(A \cup B)^{\prime \prime}$. We will show that $G_{(A \cup B)^{n}}$ is shrinkable. Shrinkability of the decomposition $G_{a}$ of $E^{2}$ implies that the nondegenerate elements of $A^{\prime \prime}$ are shrinkable in $A^{\prime \prime}$. Analogously, nondegenerate elements of $B^{\prime \prime}$ are shrinkable in $B^{\prime \prime}$. By the hypothesis that $A \cap$ $B \cap P(H)=\varnothing$, the nondegenerate elements of $A^{\prime \prime}$ and $B^{\prime \prime}$ are separated sets in $(A \cup B)^{n}$. This implies that, given an open set containing all nondegenerate elements of $(A \cup B)^{\prime \prime}$, there are two disjoint open sets in $(A \cup B)^{\prime \prime}$ such that one, $U_{A^{\prime \prime}}$, contains the nondegenerate elements of $A^{\prime \prime}$, and the other, $U_{B^{\prime \prime}}$, contains the nondegenerate elements of $B^{\prime \prime}$. Now, the required shrinking homeomorphism $f_{\epsilon}$ for all nondegenerate elements can be defined to be the composition of a homeomorphism $\left(f_{\epsilon}\right)_{A^{\prime \prime}}$ that satisfies McAuley's definition in $U_{A^{\prime \prime}}$ and agrees with a given homeomorphism $b$ off $U_{A^{\prime \prime}}$ and another homeomorphism $\left(f_{\epsilon^{\prime}}\right)^{\prime \prime}$ that satisfies the definition in $U_{B^{n}}$ and agrees with $b$ off $U_{B^{n}}$.

We will apply the following theorem (McAuley [8]) to the decomposition $G_{(A \cup B)^{n}}$ of $(A \cup B)^{\prime \prime}$.

Theorem. Suppose that $G$ is a u.s.c. decomposition of a complete metric space M. Furthermore, $H$ (the collection of all nondegenerate elements of $G$ ) is shrinkable and $M$ is locally compact at each point of $H^{*}$. Then the decomposition space is bomeomorpbic to $M$.

The decomposition $G_{(A \cup B)^{\prime \prime}}$ of $(A \cup B)^{n}$ satisfies the hypotheses of this theorem. Hence, $(A \cup B)^{\prime \prime} / G_{(A \cup B)^{n}}$ is homeomorphic to $(A \cup B)^{\prime \prime}$.

By the homeomorphisms $\phi_{A}$ and $\phi_{B}$ we carry this over to the space $(A \cup B)^{\prime}$ and find that $(A \cup B)^{\prime} / G_{(A \cup B)^{\prime}}$ is homeomorphic to $(A \cup B)^{\prime}$. (Notice that we chose to work with the decomposition of $(A \cup B)^{\prime \prime}$ instead of the decomposition of $(A \cup B)^{\prime}$, because $(A \cup B)^{\prime} \subset E^{3}$ and the shrinking in an open cover of $H_{(A \cup B)^{\prime}}$ may move nondegenerate elements out of $(A \cup B)^{\prime}$.) By the definition of the decomposition $G_{(A \cup B)^{\prime}}$, the set $A \cup B$ is homeomorphic to $(A \cup B)^{\prime} / G_{(A \cup B)^{\prime *}}$ Hence, $A \cup B$ is homeomorphic to $(A \cup B)^{\prime}$, and the theorem is proved. 
Theorem 1 may be difficult to apply because of the hypothesized conditions concerning the existence of particular $P$-lifts. Hence, the following lemma and theorem may be useful.

Lemma 2.1. Suppose that $A$ is a compact 2-manifold contained in a disk $D$ $C E^{3} / G$ and that $A$ bas a $P$-lift $A^{\prime}$. Also, suppose that $A \cap P(H)$ is 0-dimensional. Then $P \mid A^{\prime}$ is monotone.

Proof. Suppose the lemma is false. Then there is an element $g$ of $G$ such that $g \cap A^{\prime}$ is not connected. Let $s$ and $t$ be two of the components of $g \cap A^{\prime}$. For each positive integer $j$, consider the open $1 / j$ neighborhood of $P(g)$. In the intersection of $A$ with this neighborhood, let $K_{j}$ denote the component containing $P(g)$. Compactness of the $P$-lift $A^{\prime}$ implies that there exists a value $J$ of $j$ such that $s$ and $t$ lie in different components of $A^{\prime} \cap P^{-1}\left(K_{J}\right)$. Let $U_{s}$ and $U_{t}$ be the components of $A^{\prime} \cap P^{-1}\left(K_{J}\right)$ containing $s$ and $t$, respectively.

Because the set $K_{J}$ is open with respect to $A$, it is a 2-manifold. Hurewicz and Wallman $[6$, p. 48] prove that an $n$-manifold cannot be disconnected by a subset of dimension less than or equal to $n-2$. Therefore, $K_{J}-P(H)$ is connected. The map $P^{-1}$ is a homeomorphism on this set. This implies that $P^{-1}\left(K_{J}-P(H)\right)$ is connected. Also, $P^{-1}$ being a homeomorphism on the set implies that

$$
P^{-1}\left(K_{J}-P(H)\right)=A^{\prime} \cap P^{-1}\left(K_{J}-P(H)\right) \supset\left(U_{s}-H^{*}\right) \cup\left(U_{t}-H^{*}\right) \text {. }
$$

Since $U_{s}$ and $U_{t}$ are components of $A^{\prime} \cap P^{-1}\left(K_{J}\right)$ and $A^{\prime} \cap P^{-1}\left(K_{J}\right)$ contains the connected set $P^{-1}\left(K_{J}-P(H)\right)$, it must be that one of $U_{s}$ and $U_{t}$ contains no point in $P^{-1}\left(K_{J}-P(H)\right)$. Suppose $U_{s}$ contains no point of this set, and therefore, that $U_{S} \subset H^{*}$.

We note that a compact manifold can have only a finite number of components. Let $C$ be the component of $A^{\prime}$ containing $U_{s^{*}}$. If $C$ is not also the component of $A^{\prime}$ containing $U_{t}$, then $P\left(A^{\prime}\right)=A$ has fewer components than $A^{\prime}$ and this contradicts the $P$-lift requirement that $A$ be homeomorphic to $A^{\prime}$. Hence, $U_{t} \subset C$, which implies that $U_{s}$ is not all of $C$. The set $U_{s}$ is open in component $C$ and $s$ is compact, so $s$ is not all of $U_{s}$. The facts that $U_{s}$ is connected and $s$ is a component of $g \cap A^{\prime}$ imply that $U_{s}-s \not \subset g \cap A^{\prime}$. Therefore, $P\left(U_{s}-s\right) \not \subset P(g)=$ $P(s)$. But $P\left(U_{s}-s\right) \cup P(s)=P\left(U_{s}\right)$. Hence, $P\left(U_{s}\right)$ is not degenerate. The set $U_{s}$ is connected, so $P\left(U_{s}\right)$ is connected. Now, since $P\left(U_{s}\right) \subset P(H)$, the set $P\left(U_{s}\right)$ is 0 -dimensional. We now have a contradiction of the fact that any 0 -dimensional connected set is degenerate ${ }_{n}$ and this proves our lemma.

From this lemma and Theorem 1 we obtain the following:

Theorem 2 Suppose that $A$ and $B$ are compact 2-manifolds that each P-lift; $A \cup B$ is a disk $D \subset E^{3} / G ; A \cap B \cap P(H)=\varnothing$; and $(A \cup B) \cap P(H)$ is 0-dimen. sional. Then $A \cup B$ P-lifts. 
3. The condition that there exist a $P$-liftable disk $\epsilon$-homeomorphic to a given disk. As shown by the example in $\$ 1$, even when a disk does not $P$-lift, there may be a $P$-liftable disk $\epsilon$-homeomorphic to it. Theorems in this section give conditions that guarantee that such a $P$-liftable disk exists.

Theorem 3. Suppose that $E^{3} / G$ is bomeomorphic to $E^{3}, P(H)$ is countable, and $D$ is a disk in $E^{3} / G$. Furthermore, suppose that there is a P-liftable, compact manifold $C$ contained in $D$ and such that $\mathrm{Cl}(D-C)$ is a manifold and $C \cap$ $\mathrm{Cl}(D-C)$ misses $P(H)$. Then, given $\epsilon>0$, there is a disk $D_{\epsilon}^{1}$ such that

(1) $D_{\epsilon}^{1}$ is $\epsilon$-bomeomorphic to $D$,

(2) the e-bomeomorphism is the identity on $C$, and

(3) $D_{\epsilon}^{1}$ P.lifts.

Proof. Let $F$ denoce the manifold $\mathrm{Cl}(D-C)$. In $E^{3} / G$ approximate $F$ by a manifold $F_{p}$ that is $\epsilon / 2$-homeomorphic to $F$, misses $C$ off $F \cap C$, agrees with $F$ on $F \cap C$, and is locally polyhedral on $F_{p}-C$. (This can be done using Bing's Approximation Theorem [2].) There is a locally finite triangulation $T$ for $F_{p}-C$ (Hudson [s]). Let $v_{1}, v_{2}, v_{3}, \cdots$ be the vertices of $T$. By moving these vertices slightly, we will obtain a new complex $F_{0}$ close to $F_{p}$, missing $P(H)$, and such that $C \cup F_{0}$ is a disk.

First, we adjust $v_{1}$. A new vertex $v_{1}^{\prime}$ will be chosen so $d\left(v_{1}, v_{1}^{\prime}\right)<\epsilon / 2$ and the cone of $v_{1}^{\prime}$ over $\mathrm{Lk} v_{1}$ is a disk $E_{1}$ that misses $F_{p}-\mathrm{St} v_{1}, C$, and $P(H)$. ( $\mathrm{Lk}$ and St denote link and star, respectively.) Such a point $v_{1}^{\prime}$ can be found by the following method. Let $r$ be a point in general position with respect to the vertices of $T$ and close enough to $v_{1}$ that any point $p$ in the segment $\left[v_{1}, r\right]$ satisfies all the conditions except possibly the one concerning the cone missing $P(H)$. Note that the interiors of the cones from two points $p_{1}$ and $p_{2}$ in $\left[v_{1}, r\right]$ are dis joint open disks. There is an uncountable number of these mutually disjoint open disks. Since there is only a countable number of points in $P(H)-P(C)$, there must exist a point $v_{1}^{\prime} \in\left[v_{1}, r\right]$ satisfying all the conditions.

Inducting on the subscript $i$, each vertex $v_{i}$ can be moved to satisfy the above conditions for the new cone and to be within $\epsilon / 2^{i}$ of its original position. With this distance requirement, the set resulting from moving all vertices is a manifold $F_{0}$ such that $C \cup F_{0}$ is a disk and $C \cap F_{0}=C \cap F_{p}=C \cap F$. Also, $F_{0}$ is $\epsilon / 2$-homeomorphic to $F_{p}$. Since $F_{p}$ is $\epsilon / 2$-homeomorphic to $F$, the disk $C \cup F_{0}$ is $\epsilon$-homeomorphic to the given disk $D$. Let $C \cup F_{0}$ be denoted by $D_{\epsilon}^{1}$.

We use Theorem 2 to show that $D_{\epsilon}^{1} P$-lifts. The manifolds $C$ and $F_{0}$ are the sets $A$ and $B$, respectively, of The orem 2. The condition $F_{0} \cap C \cap P(H)=$ $\varnothing$ is satisfied. Since $P(H)$ is countable, it is 0 -dimensional. We have satisfied the hypotheses of Theorem 2, so it gives us the conclusion that $D_{\epsilon}^{1} P$-lifts.

Theorem 4. Suppose $E^{3} / G$ is bomeomorphic to $E^{3}$ and that $P(H)$ is count- 
able. Given a disk $D$ in $E^{3} / G$ and $\epsilon>0$, there is a disk $D_{\epsilon}^{t}$ that is $\epsilon$-bomeomorphic to D, tame, and P.lifts to a tame disk.

Proof. There is a polyhedral disk $D_{p}$ that is $\epsilon / 2$-homeomorphic to $D$ (Bing [2]). Since $D_{p}$ is polyhedral, in a neighborhood $N$ of $D_{p}$ there is a set homeomorphic to $D_{p}^{p} \times[0,1]$. Let $\theta: D_{p} \times[0,1] \rightarrow N$ be the homeomorphism with $\theta$ being the identity on $D_{p} \times\{0\}$. Notice that for $s_{1}, s_{2} \in[0,1]$ where $s_{1} \neq s_{2}$, the disks $\theta\left(D_{p} \times\left\{s_{1}\right\}\right)$ and $\theta\left(D_{p} \times\left\{s_{2}\right\}\right)$ are disjoint. Since for each $p \in D_{p}$ the point $\theta(p \times\{0\})$ is $p$, there is some $\delta>0$ such that $d(\theta(p \times\{s\}), p)<\epsilon / 2$ for each $s \epsilon$ $[0, \delta]$. Letting $D_{s}$ denote the $\operatorname{disk} \theta\left(D_{p} \times\{s\}\right)$, we have that $D_{s}$ is $\epsilon / 2$-homeomorphic to $D_{p}$. Let $C$ denote the collection $\left\{D_{s} \mid s \in[0, \delta]\right\}$. This is an uncountable collection of disjoint disks, each $\epsilon / 2$-homeomorphic to $D_{p}$.

For every $s \in[0, \delta)$ the disk $D_{s}$ is tame because it is collarable. At most a countable number of members of $C$ can contain points of the countable set $P(H)$. Hence, we have an uncountable subcollection $C_{1} \subset C$ of tame disks that contain no points of $P(H)$. Now $P^{-1}$ is a homeomorphism on this set and, hence, each disk in $C_{1} P$-lifts. It remains to be shown that the $P$-lift of each element of $C_{1}$ is tame. For this we will apply the following theorem (Armentrout [1]):

Theorem. Suppose that $M$ is a 3-manifold with boundary and $G$ is a cellular decomposition of $M$ such that $M / G$ is a 3-manifold with boundary.. Suppose $K$ is a 2-manifold with boundary in $M$ such that $K$ misses. $H$. Then $P(K)$ is tame in $M / G$ if and only if $K$ is tame in $M$.

For $M / G$ we use a 3-ball, $B$, containing $C_{1}^{*}$ in its interior and such that its boundary misses $P(H)$. For $M$ we use $P^{-1}(B)$ and we let $K$ be any member of the collection $C_{1}$, To satisfy the hypotheses we still need to know that $G$ is a cellular decomposition. This we obtain by citing the following theorem of Kwun [7].

Theorem. If $E^{3 / G}$ is bomeomorpbic to $E^{3}$ and $H$ is countable, then eacb element of $H$ is cellular.

The conclusion of Armentrout's theorem now implies that any member of $C_{1}$ satisfies the requirements for $D_{\epsilon}^{t}$.

Theorem 5. Suppose that $H$ is definable by 3-cells, $D$ is a disk in $E^{3} / G$ and $C$ is a compact manifold in $D$ such that $(\mathrm{Bd} C) \cap \mathrm{Cl} P(H)=\varnothing$ and $C$ P-lifts. Then, given $\epsilon>0$, there is a disk $D_{\epsilon}^{1}$ such that

(1) $D_{\epsilon}^{1}$ is $\epsilon$-bomeomorpbic to $D$,

(2) the $\epsilon$-bomeomorphism is the identity on $C$, and

(3) $D_{\epsilon}^{1} P$-lifts.

Proof. As in the proof of Theorem 3, we will use Theorem 2 to add a P-liftable and a liftable manifold. It is possible that $D-C$ contains an infinite number of components, and, hence, that $\mathrm{Cl}(D-C)$ is not a manifold. Since there is 
a positive distance between $\mathrm{Bd} C$ and $\mathrm{Cl} P(H)$, only a finite number of these components can contain points of $P(H)$. Let $\hat{C}$ be the union of $C$ and those components of $D-C$ that contain no points of $P(H)$. The sets we will add by means of Theorem 2 are $\hat{C}$ and a set that will approximate $\mathrm{Cl}(D-\hat{C})$.

We wish to know that $E^{3} / G$ is homeomorphic to $E^{3}$. This is immediate from the following result of Harrold [3]. Note that definability by 3-cells implies his hypotheses.

Theorem. If $G$ is a monotone decomposition of $S^{3}$ such that each point of $\mathrm{Cl} P(H)$ bas arbitrarily small neighborboods in $S^{3} / G$ with boundaries that are 2 spheres dis joint from $\mathrm{Cl} P(H)$, then $S^{3} / G$ is a 3-sphere.

Let $H_{D-C}$ denote the subset of $H$ that projects into $D-\hat{C}$. Let $\mathcal{U}$ be an open (in $\left.E^{3} / G\right)$ cover of $\mathrm{Cl} P\left(H_{D-C}\right)$ such that, for each $P(g) \in \mathrm{Cl} P\left(H_{D-C}\right)$, there is a $U_{g} \in \mathcal{U}$ contained in the $\epsilon / 2$-neighborhood of $P(g)$ and missing $\hat{C}$. Then, for each $U_{g} \in \mathcal{U}$, the set $P^{-1}\left(U_{g}\right)$ is open and contains $g$. Since $H$ is definable by 3 -cells, there is a component $B_{8}$ of some element of the defining sequence such that $g \subset B_{g} \subset P^{-1}\left(U_{g}\right)$. This gives us an open cover, $\left\{\right.$ Int $\left.B_{g}\right\}$ $g \in \mathrm{Cl} H_{D-C}$ \}, of the compact set $\stackrel{8}{\mathrm{C} l} H_{D-C}^{*}$. Suppose that $\left\{\right.$ Int $B_{1}$, Int $B_{2}, \cdots$, Int $\left.B_{k}\right\}$ is a minimal finite subcover. Notice that $\left\{B_{1}, B_{2}, \cdots, B_{k}\right\}$ is a set of disjoint 3-cells, because each $B_{j}$ is a component of some defining manifold. Since $\mathrm{Bd} B_{j}$ is a 2-sphere missing $H^{*}$, its image $P\left(\mathrm{Bd} B_{j}\right)$ is also a 2-sphere. Because $E^{3} / G$ is homeomorphic to $E^{3}$, we now have that $P\left(\mathrm{Bd} B_{j}\right)$ bounds a 3cell, namely, $P\left(B_{j}\right)$. Hence, $\left\{P\left(B_{j}\right) \mid j=1,2, \cdots, k\right\}$ is a set of disjoint 3-cells that cover $C l P\left(H_{D-C}\right)$. We will find our new disk $D_{\epsilon}^{1}$ in the union of these $3-$ cells and $D$.

Consider one 3-cell $A_{j}=P\left(B_{j}\right)$. Since $A_{j} \cap \mathrm{Cl} P(H) \subset$ Int $A_{j}$, there is a

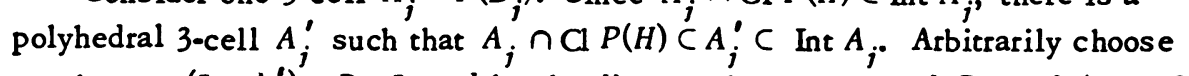
a point $x \in\left(\text { Int } A_{j}^{\prime}\right)^{j}-D$. Let $d$ be the distance between $x$ and $D \cup \operatorname{Bd} A_{j}$, and $N$ be the closed $d / 2$ neighborhood of $x$. Then $N$ is a tame 3 -cell inside the tame 3-cell $A_{j}^{\prime}$ and $N$ misses $D$. There is a homeomorphism $b_{j}: E^{3} / G \rightarrow E^{3} / G$ such that $b_{j}\left(A_{j}^{\prime}\right)=N$ and, for any $y \in E^{3} / G$ - Int $A_{j}, b_{j}(y)=y$. Notice that, for each $z \in P(H) \cap A_{j}$, the image $b_{j}(z)$ misses $D$. Therefore, $\left(b_{j}^{-1}(D)\right) \cap z=\varnothing$. Of course, $b_{j}^{-1}$ is the identity on $D-A_{j}$. It is an $\epsilon$-homeomorphism since the diameter of $A_{j}$ is less than $\epsilon$.

Let $b=b_{k} b_{k-1} \cdots b_{1}$. It is an $\epsilon$-homeomorphism. Since $\operatorname{Cl} P(H) \cap\left(\bigcup_{j} A_{j}\right)$ $\subset \cup_{j} A_{j}^{\prime}, \mathrm{Cl} b^{-1}(D-\hat{C})$ misses $P(H)$ and $\mathrm{Cl}^{-1}(D-\hat{C})$ lifts. By our choice of the cover $\mathcal{U}$, we know that Int $b^{-1}(D-\hat{C})$ does not intersect $\hat{C}$ and that $b^{-1}$ is the identity on Bd $\hat{C}$. Therefore, $\hat{C} \cup \mathrm{Cl}^{-1}(D-\hat{C})$ is a disk. Denote this disk by $D_{\epsilon}^{1}$. We will now use Theorem 2 to show that $D_{\epsilon}^{1}$ does $P$-lift. Our decomposition is 0-dimensional because it has a defining sequence. For the 2manifolds $A$ and $B$ we use, respectively, $\hat{C}$ and $C l b^{-1}(D-\hat{C})$.

In Theorems 3 and 5 consider weakening the hypotheses on the decomposition 
spaces in the following manner. Suppose that $G$ is a $(2,1)$ toroidal decomposition (for this terminology, see Sher [12]) with an uncountable number of elements. Then in $E^{3} / G$ there does exist a disk $D$ that has no $P$-liftable disk $D_{\epsilon}$ that is $\epsilon$-homeomorphic to $D$. This example and related ones will be discussed in a future paper [16]. Also, see Woodruff [14], [15], in which a similar example is discussed.

4. The condition that the $P$-liftable disk $D_{\epsilon}$ agree with $D$ on the singular points. In the following theorem we add hypotheses that make it possible to push a $P$-liftable disk $D_{\epsilon}$ to the singular points of the given disk $D$ and to reta in the $P$-lift property.

Theorem 6. Suppose that $G$ is monotone, $\mathrm{Cl} P(H)$ is 0-dimensional, and $\mathrm{Cl} H^{*}$ is 1-dimensional. Let $M \subset E^{3 / G}$ be a compact 2-manifold with or without boundary and let the collection $H_{M}$ of nondegenerate elements that project into $M$ be cont inuous. Suppose that, for any $\epsilon>0$, there is a manifold $M_{\epsilon}^{1}$ that P.lifts, is $\epsilon-$ bomeomorphic to $M$, and satisfies the containment: $M_{\epsilon}^{1} \cap P(H) \subset M \cap P(H)$. Then we conclude that for each $\epsilon>0$ there is a manifold $M_{\epsilon}$ that $P$-lifts, is $\epsilon-$ bomeomorpbic to $M$, and also satisfies the equality: $M_{\epsilon} \cap \stackrel{\epsilon}{(H)}=M \cap P(H)$.

Proof. Let $\epsilon>0$ be given. Then, for $\eta=\epsilon / 4$, there exists a manifold $M_{\eta}^{1}$ with the hypothesized properties with respect to $\eta$. For simplicity of notation, let us denote this manifold by $K_{0}$ and its $P$-lift by $K_{0}^{\prime}$. Let $\theta: M \rightarrow K_{0}$ be an $\epsilon / 4$-homeomorphism. For each $p \in M \cap \mathrm{Cl} P(H)$, let $B_{p}$ be the open $\epsilon / 2$-neighborhood of $p$. Notice that each $P^{-1}\left(B_{p}\right)$ intersects $K_{0}^{p}$. In $K_{0}^{\prime} \cup\left(U_{p} P^{-1}\left(B_{p}\right)\right)$ we plan to construct a new manifold $M_{\epsilon}^{0}$, whose projection will satisfy the conclusion of the theorem. The construction will consist of pushes of subdisks of $K_{0}^{\prime}$ forming protrusions that we will refer to as "fingers", and successive stages of finger formation making a "treelike" structure. As fingers grow on previous fingers, the sets will reach out toward points that we wish to add in order to satisfy the equality in the conclusion. When we push out a finger, we will have a homeomorphism in the domain between the old and new manifolds. To ensure that their projections will be homeomorphic, the construction will not add more than one point of any element of $H$, and will add no points of elements of $H$ already intersecting the manifold $K_{0}^{\prime}$. We will describe the construction in such a way that it will be possible to prove that the composition of the infinite sequence of the homeomorphisms in the image space is an $\epsilon / 2$-homeomorphism. This composed with $\theta$ will give us our desired $\epsilon$-homeomorphism.

From the sets $P^{-1}\left(B_{p}\right)$ arbitrarily choose a finite open covering of $\mathrm{Cl} H_{M}^{*}$. Let $L$ be the Lebesgue number for this corer.

In the first stage of finger formation we will use the elements of $H_{M}$ that do not intersect $K_{0}^{0}$. We denote them by $H_{M}^{0}$. In $\left(H_{M}^{0}\right)^{*}$ choose a finite set of points, denoted by $\left\{z_{1}^{1}, z_{1}^{2}, \cdots, z_{1}^{k}\right\}$, that are $L / 2$ dense in $\left(H_{M}^{0}\right) *$. It follows from the definition of the Lebesgue number that the $L / 4$-neighborhood of the point $z_{1}^{1}$ lies 
in at least one set, denoted $P^{-1}\left(B_{p_{1}}\right)$, of the chosen sets. Define $T_{1}^{1}=$ $\left(P^{-1}\left(B_{p_{1}}\right)\right)-\mathrm{Cl} H^{*}$. In the $L / 4$-neighborhood of $z_{1}^{1}$ choose a point $x_{1}^{1}$ that is arcwise accessible in $T_{1}^{1}$. (Notice that $x_{1}^{1} \notin \mathrm{Cl} H^{*}$.)

We wish to have a point $y_{1}^{1}$ in $T_{1}^{1} \cap K_{0}^{\prime}$ such that $y_{1}^{1}$ is arcwise accessible in $T_{1}^{1}$ to the point $x_{1}^{1}$. The following argument shows the existence of $y_{1}^{1}$. For $p_{1}$ used in defining $B_{p_{1}}$, we have $d\left(\mathrm{Bd} B_{p_{1}}, p_{1}\right)=\epsilon / 2$ and $d\left(K_{0}, p_{1}\right)<\epsilon / 4$. Hence, there is a subdisk of $K_{0}$ contained in $B_{p_{1}}$. In fact, since $\mathrm{Cl} P(H)$ does not contain $K_{0}$, there is a subdisk of $K_{0}-\mathrm{Cl} P(H)$ in $B_{p_{1}}$. Equivalently, there is a subdisk of $K_{0}$ in $B_{p_{1}}-\mathrm{Cl} P(H)$. Hence, there is a subdisk of $K_{0}^{\prime}$ in $T_{1}^{1}$. Now, by hypothesis, $\mathrm{Cl} H^{*}$ is 1-dimensional. Since $P^{-1}\left(B_{p_{1}}\right)$ is an open connected subset of $E^{3}$, no 1-dimensional set can separate it (Hurewicz and Wallmann $\left[6\right.$, p. 48]). Hence, $T_{1}^{1}$ is connected. Now $T_{1}^{1}$ is again an open connected subset of $E^{3}$, so if it is separated by $K_{0}^{\prime}$, each subset of $K_{0}^{\prime}$ separating it must be 2 -dimensional. Hence, each component of $T_{1}^{1}-K_{0}^{\prime}$ contains a 2-dimensional subset of $K_{0}^{\prime}$ in its boundary. We now cite a theorem in Hocking and Young [4, p. 119] that states that in a locally connected and locally arcwise connected space $S$, the set of all points on the boundary of an open set $W$ that are accessible from $W$ is dense in the boundary of $W$. This implies that accessible points in the boundary of $T_{1}^{1}-K_{0}^{\prime}$ are dense in the boundary, and hence, dense in $T_{1}^{1}$ $\cap K_{0}^{\prime}$. This implies that there exists a point $y_{1}^{1}$.

Let $a$ be an arc in $T_{1}^{1}$ from $x_{1}^{1}$ to $y_{1}^{1}$. (See Figure 1.) In $T_{1}^{1} \cap K_{0}^{1}$ there is a disk $D$ containing $y_{1}^{1}$ in its interior and with diameter less than $L$. By covering each point of Int $a$ with an appropriately small open neighborhood, we can fatten Int $\alpha$ to obtain an open ball $A$ satisfying

(1) Int $a \subset A$,

(2) $K_{0}^{\prime} \cap \mathrm{Cl} A=D \subset \mathrm{Bd} A$, and

(3) $H^{*} \cap \operatorname{Bd} A=\varnothing$.

We use $\mathrm{Cl}((\operatorname{Bd} A)-D)$ to replace the disk $D$. This is the finger. We call $D$ its base and $x_{1}^{1}$ its tip.

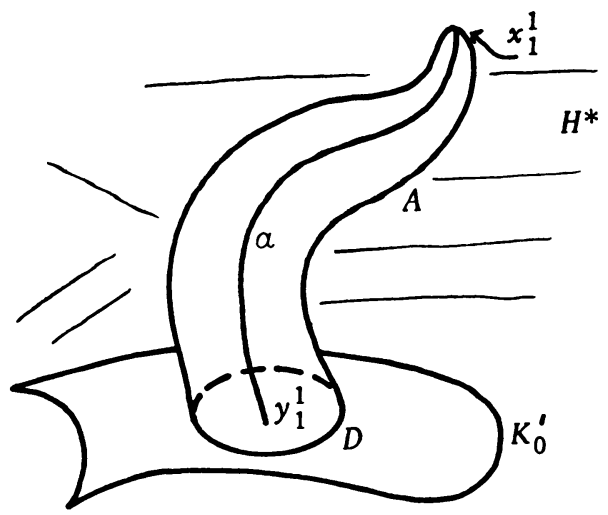

Figure 1 
We have described the formation of one finger at the first stage. Using the points $z_{1}^{1}, z_{1}^{2}, \ldots, z_{1}^{k}$, it is possible to form a set of $k$ such disjoint fingers. This is the first stage of the construction. Call the resulting manifold $K_{1}^{\prime}$ and its projection $K_{1}$. Let $b_{1}$ be a homeomorphism taking $K_{0}^{\prime}$ onto $K_{1}^{\prime}$ by the finger construction.

At each successive stage, new fingers grow on previously formed fingers. This growth must be controlled in the following ways. (1) New finger size must go to zero; (2) sets growing from different fingers must not be approaching the same point in the domain; and (3) sets grow ing from different fingers must not be approaching different points in the same nondegenerate element.

The base of each finger at the $i$ th stage will be contained in one $i-1$ stage finger-the "parent" finger. Each finger will have a "territory" in which it is permitted to grow. Territories will be defined so that at each stage the territories of new fingers being formed will be separated sets, and the territory of each new finger will lie in that of its parent. Let $T_{i}^{j}$ denote the territory of the $j$ th finger at the $i$ th stage. For $j \neq k$, no nondegenerate element will intersect both $T_{i}^{j}$ and $T_{i}^{k}$. Conditions (2) and (3) will be satisfied if $P\left(T_{i}^{j}\right) \cap P\left(T_{i}^{k}\right)=\varnothing$.

For the first step in defining territories we modify at each stage the subset of $\left(H_{M}^{0}\right) *$ toward which fingers grow. We work first in the second stage. Though the points $x_{1}^{1}, x_{1}^{2}, \ldots, x_{1}^{k}$ are all used for a single stage, the definition of the modified subset for the second stage is made in $k$ steps rather than one. Let

$$
\left(H_{M}^{0}\right)^{1}=\left\{\left.g \cap N_{2 \lambda}\left(x_{1}^{1}\right)\right|_{g} \in H_{M}^{0}, \lambda=d\left(g, x_{1}^{1}\right)\right\},
$$

where $N_{a}(B)$ denotes the a-neighborhood of a set $B$. In Figure 2 some elements of $H_{M}^{0}$ are shown with those portions that are not also in $\left(H_{M}^{0}\right)^{1}$ crossed out. Observe that no element is totally deleted.

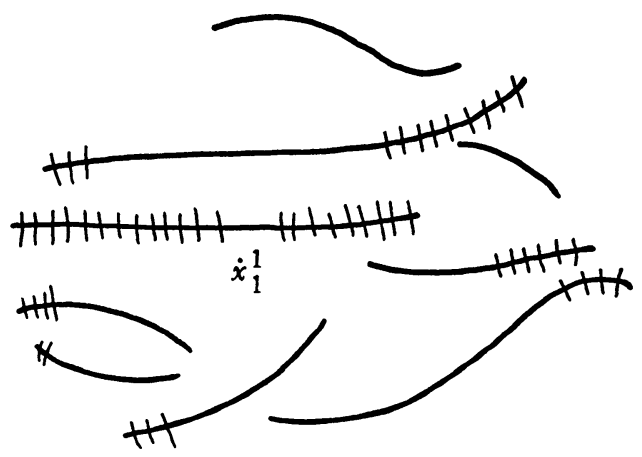

Figuire 2 
Define

$$
\begin{aligned}
\left(H_{M}^{0}\right)^{2} & =\left\{g \cap N_{2 \lambda}\left(x_{1}^{2}\right) \mid g \in\left(H_{M}^{0}\right)^{1}, \lambda=d\left(g, x_{1}^{2}\right)\right\} \\
& \vdots \\
\left(H_{M}^{0}\right)^{k} & =\left\{g \cap N_{2 \lambda}\left(x_{1}^{k}\right) \mid g \in\left(H_{M}^{0}\right)^{k-1}, \lambda=d\left(g, x_{1}^{k}\right)\right\}=H_{M^{\circ}}^{1}
\end{aligned}
$$

The sets $\left(H_{M}^{0}\right)^{j}$ each retain the property of being continuous collections.

For the points near the tips of fingers at the second stage choose in $\left(H_{M}^{1}\right) *$ a finite set of points denoted by $\left\{z_{2}^{1}, z_{2}^{2}, \ldots, z_{2}^{m}\right\}$ that are $L / 4$ dense in $\left(H_{M}^{1}\right) *$.

The following theorem is slightly modified from one that is proved in Hurewicz and Wallman $[6$, p. 54].

Theorem. Let $X$ be a 0 -dimensional space and $U_{1}, U_{2}, \ldots, U_{,}$be an open covering of $X_{0}$. Then there exists a refining covering by disjoint open sets $V_{1^{\prime}}$, $v_{2}, \ldots, V_{r}$

This theorem must be used in $E^{3} / G$ where $\mathrm{PCl}\left(H_{M}\right)$ is 0-dimensional. We will start with an open covering of a set in $E^{3}$, then find a related open covering of the 0 -dimensional set in $E^{3} / G$, apply the theorem there, and then return to $E^{3}$ with disjoint sets that cover $\mathrm{Cl}\left(H_{M}^{1}\right) *$.

Let us require that the second stage territory $T_{2}^{j}$ be contained in the intersection of the $L$-neighborhood of $z_{2}^{j}$ and the set $P^{-1}\left(B_{p}\right)$ that is the parent of this $j$ th finger. Denote this intersection by $U_{2}^{j}$. Since the limiting set of our construction must contain a point in every $g \in H_{M}$, we must make $\left\{P\left(T_{2}^{j}\right)\right\}$ cover $P\left(H_{M}^{1}\right)$. Note that although $\left\{P\left(U_{2}^{j}\right)\right\}$ covers $P\left(H_{M}^{M}\right)$, it is not necessarily an open cover in the decomposition space.

Since $H_{M}^{1}$ is continuous, it is lower semicontinuous. For each $j$ this implies that from the open with respect to $E^{3}$ set $U_{2}^{j}$ we get the open with respect to $\mathrm{Cl}\left(H_{M}^{1}\right) *$ set $V_{2}^{j}=\left\{y \in g \mid g \cap U_{2}^{j} \neq \varnothing, g \in \mathrm{Cl}_{M}^{1}\right\}$. Each $V_{2}^{j}$ is an inverse set, so $\left\{P\left(V_{2}^{j}\right)\right\}$ must be an open with respect to $P\left(C l H_{M}^{1}\right)$ cover of $P\left(H_{M}^{1}\right)$. Using the above theorem, the 0 -dimensionality of $P\left(H_{M}^{1}\right)$ implies that there is a refining cover $\left\{W_{2}^{j}\right\}$ of disjoint sets such that for each $j$, the set $W_{2}^{j} \subset P\left(V_{2}^{j}\right)$ is both open and closed with respect to $P\left(C l H_{M}^{1}\right)$. (Notice that if, for some $j$, the point $z_{2}^{j}$ $\notin \mathrm{Cl}\left(H_{M}^{1}\right) *$, then it is possible, but not necessarily true, that $W_{2}^{j}$ is empty. In this case the corresponding finger will have no territory and will not exist.) The set $P^{-1}\left(W_{2}^{j}\right)$ is closed with respect to $\mathrm{Cl}\left(H_{M}^{1}\right) *$. Returning to $E^{3}$, we see that $P^{-1}\left(W_{2}^{j}\right)$ is also closed there. The finite number of disjoint closed sets in $\left\{P^{-1}\left(W_{2}^{j}\right) \mid j=1,2, \ldots, m\right\}$ can be covered by disjoint open sets in $E^{3}$. Let $Y_{2}^{j}$ denote the open set containing $P^{-1}\left(W_{2}^{j}\right)$. Now the sets $\left\{U_{2}^{j} \cap Y_{2}^{j} \mid j=1,2, \ldots\right.$, $m\}$ are almost the desired "territory" sets. They do contain all necessary points for adding and are separated and open with respect to $E^{3}$. It is possible that, for some values of $j$, the set $U_{2}^{j} \cap Y_{2}^{j}$ is not connected or does not intersect 
$K_{1}^{\prime}$. Let $\left\{\beta_{2}^{j} \mid j=1,2, \ldots, m\right\}$ be a set of disjoint arcs such that each arc $\beta_{2}^{j}$ is contained in $U_{2}^{j}-\mathrm{Cl} H^{*}$ and $\beta_{2}^{j}$ connects all components of $\left(U_{2}^{j} \cap Y_{2}^{j}\right) \cup K_{1}^{\prime}$. The arcs can be thickened to disjoint open tubular neighborhoods $\gamma_{2}^{j}$ that do not separate $U_{2}^{k}$ for any $k \neq j$ and miss $\mathrm{Cl} H^{*}$. The territory of the $j$ th finger is $\left(\left(U_{2}^{j} \cap Y_{2}^{j}\right) \cup \gamma_{2}^{j}\right)-\bigcup_{k=1 ; k \neq j}^{m} \gamma_{2}^{k}$.

Now that the territories are defined and the points $z_{2}^{j}$ have been chosen, we need only to construct the fingers for the second stage. In the $L / 8$-neighborhood of each $z_{2}^{j}$ choose a point $x_{2}^{j}$ that lies in the territory $T_{2}^{j}$. In the territories, choose the arcs $a$, fatten them into balls, and replace subdisks of $K_{1}^{\prime}$ with fingers. By the definition of the territory, each finger will have set diameter no more than $L$. Choose the base so that it is the image under $b_{1}$ of a set in $K_{0}^{\prime}$ having diameter no more than $L / 2$. We now have constructed $K_{2}^{\prime}$. Let $b_{2}$ be the homeomorphism resulting from the finger construction taking $K_{1}^{\prime}$ onto $K_{2}^{\prime}$.

For the $i$ th stage, modify $H_{M}^{i-1}$ in the finite number of steps corresponding to the fingers used in the $i-1$ stage. In the new set $\left(H_{M}^{i}\right) *$ choose a finite set of points $\left\{z_{i}^{j}\right\}$ that are $L /\left(2^{i}\right)$ dense. Choose disjoint territories $T_{i}^{j}$ by using the continuity of $H_{M}^{i}$. The diameter of $T_{i}^{j}$ is less than $L /\left(2^{i-1}\right)$. In $T_{i}^{j}$ choose $y_{i}^{j}$ in $K_{i-1}^{\prime}$ and $x_{i}^{j}$ in the $L /\left(2^{i+1}\right)$-neighborhood of $z_{i}^{j}$. Connect these by an arc in $T_{i}^{j}$. For the base of the finger use a disk that is the image under $b_{i-1} b_{i-2}$ $\cdots b_{1}$ of a set in $K_{0}^{\prime}$ having diameter no more than $L /\left(2^{i}\right)$. There is a homeomorphism $b_{i}$ taking $K_{i-1}^{\prime}$ onto $K_{i}^{\prime}$ by the finger construction.

It can be shown that this total construction produces a manifold $M_{\epsilon}$ that satisfies the conclusions of the theorem.

From Theorems 3, 5, and 6 we immediately get the next two theorems.

Theorem 7. Suppose that $E^{3 / G}$ is bomeomorphic to $E^{3} ; H$ is countable; $\mathrm{Cl} P(H)$ is 0 -dimensional; and that, for each $g \in H, \operatorname{dim} g=1$. Let $D$ be a disk contained in $E^{3} / G$ and let the collection of nondegenerate elements that project into $D$ be continuous. Then, for any $\epsilon>0$, there is anotber disk $D_{\epsilon}$ that $P$-lifts, is $\epsilon$-bomeomorphic to $D$, and sat isfies the equality $D_{\epsilon} \cap P(H)=D \cap P(H)$.

Theorem 8. Suppose that $H$ is definable by 3-cells and $\mathrm{Cl} H^{*}$ is 1-dimensional. Let $D$ be a disk in $E^{3} / G$ and let the collection of nondegenerate elements that project into $D$ be continuous. Then, for any $\epsilon>0$, there is another disk $D_{\epsilon}$ that $P$-lifts, is $\epsilon$-bomeomorpbic to $D$, and satisfies the equality $D_{\epsilon} \cap P(H)=D \cap P(H)$.

If in Theorems 3 and 5 we use $C=\varnothing$, then the $D_{f}^{1}$ that we construct in the proof actually lifts, rather than only $P$-lifts. We are led to wonder whether, when there is a disk $D$ that $P$-lifts, there is always, for any $\epsilon>0$, another disk $D_{\epsilon}$, which is $\epsilon$-homeomorphic to $D$ and actually lifts. The following example shows that this is not true.

Let the only nondegenerate element of $G$ be a $\theta$-shaped curve. The $P$-lift 
$D^{\prime}$ is chosen to contain in its interior the middle arc of the $\theta$. No other points of the $\theta$ intersect $D^{\prime}$. There exists an $\epsilon>0$ such that there can be no $D_{\epsilon}$ that is $\epsilon$-homeomorphic to $P\left(D^{\prime}\right)$ and has a true lift.

\section{REFERENCES}

1. Steve Armentrout, Concerning cellular decompositions of 3-manifolds with boundary, Trans. Amer. Math. Soc. 137 (1969), 231-236. MR 38 \#224.

2. R. H. Bing, Approximating surfaces with polyhedral ones, Ann. of Math. (2) 65 (1957), 456-483. MR 19, 300.

3. O. G. Harrold, $\mathrm{J} \mathrm{r}$, A sufficient condition that a monotone image of the 3-sphere be a topological 3-sphere, Proc. Amer. Math. Soc. 9 (1958), 846-850. MR 21 \#2223.

4. John G. Hocking and Gail S. Young, Topology, Addison-Wesley, Reading, Mass., 1961. MR 23 \#A2857.

5. J. F. P. Hudson, Piecewise linear topology, Benjamin, New York and Amsterdam, 1969. MR $40 \# 2094$.

6. Witold Hurewicz and Henry Wallman, Dimension theory, Princeton Math. Series, vol. 4, Princeton Univ. Press, Princeron, N. J., 1941; rev. ed, 1948, p. 48. MR 3, 312.

7. Kyung Whan Kwun, Upper semicontinuous decompositions of the n-sphere, Proc. Amer. Math. Soc. 13 (1962), 284-290. MR 25 \#3512.

8. Louis F. McAuley, Upper semicontinuous decompositions of $E^{3}$ into $E^{3}$ and generalizations to metric spaces, Topology of 3-Manifolds and Related Topics (Proc. Univ. of Georgia Inst., 1961), Prentice-Hall, Englewood Cliffs, N. J., 1962, pp. 21-26. MR 25 $\# 4502$

9. Lifting disks and certain light open mappings, Proc. Nat. Acad. Sci. U. S. A. 53 (1965), 255-260. MR 30 \#2473.

10. Louis F. McAuley and Patricia Tulley, Lifting cells for certain light open mappings, Math. Ann. 175 (1968), 114-120. MR 36 \#3324.

11. R. L. Moore, Concerning upper semi-continuous collections of continua, Trans. Amer. Math. Soc. 27 (1925), 416-428.

12. R. B. Sher, Toroidal decompositions of E3, Fund. Math. 61 (1967/68), 225-241. MR 37 \#05.

13. L. C. Siebenmann, Approximating cellular maps by homeomorphisms, Topology 11 (1972), 271-294.

14. Edythe P. Woodruff, Concerning the condition that a disk in $E 3 / G$ be the image of a disk in E3, Proc. First Conf. on Monotone Mappings and Open Mappings (SUNY at Binghamton, Binghamton, N..Y., 1970), State Univ. of New York at Binghamton, Binghamton, N. Y., 1971, pp. 390-417. MR $43 \# 4010$.

15. Concerning the condition that a disk in $E 3 / G$ be the image of a disk in E3, Doctoral Dissertation, SUNY/Binghamton, 1971.

16. - Examples of disks in E3/G which can not be approximated by P-liftable disks (to appear).

DEP ARTMENT OF MATHEMATICS, STATE UNIVE RSITY OF NEW YORK AT BINGHAMTON, BINGHAMTON, NEW YORK 13901

DEPARTMENT OF MATHEMATICS, TRENTON ST ATE COLLEGE, TRENTON, NEW JERSEY 08625 (Current address) 